\title{
Une approche pour la comparaison, du point de vue fonctionnement hydraulique, de propositions d'extension d'un réseau d'assainissement
}

\section{An approach to compare, from the hydraulic operation viewpoint, propositions of extension of an urban storm drainage network}

\author{
O. Blanpain, A. Karnib, J. Al-Hajjar et D. Boissier
}

Volume 11, numéro 2, 1998

URI : https://id.erudit.org/iderudit/705306ar

DOI : https://doi.org/10.7202/705306ar

\section{Aller au sommaire du numéro}

\section{Éditeur(s)}

Université du Québec - INRS-Eau, Terre et Environnement (INRS-ETE)

\section{ISSN}

0992-7158 (imprimé)

1718-8598 (numérique)

\section{Découvrir la revue}

Citer cet article

Blanpain, O., Karnib, A., Al-Hajjar, J. \& Boissier, D. (1998). Une approche pour la comparaison, du point de vue fonctionnement hydraulique, de propositions d'extension d'un réseau d'assainissement. Revue des sciences de l'eau / Journal of Water Science, 11(2), 241-254. https://doi.org/10.7202/705306ar

\section{Résumé de l'article}

Les outils informatiques développés dans le cadre de la conception des réseaux d'assainissement permettent de concevoir plusieurs solutions de réseaux pour un même problème. Il revient ensuite au décideur de choisir quelle solution retenir. Le problème qui se pose alors est la comparaison des propositions selon des critères économiques, écologiques, de fonctionnement, de maintenance, .... La difficulté vient du fait que certains de ces critères sont difficiles à évaluer. Nous présentons dans cet article une méthode permettant d'évaluer l'un de ces critères : le fonctionnement global d'un réseau d'assainissement suite à l'extension de ce dernier. Cette méthode est basée sur des simulations hydrauliques. Or ces simulations donnent des informations en chaque noeud du réseau (histogrammes de vitesses, débits ou hauteurs de charge) et non une indication sur la qualité de fonctionnement du réseau dans son ensemble. Il nous a donc fallu élaborer une technique d'agrégation permettant de passer de l'élément isolé (le tronçon) à l'ensemble organisé (le réseau). Cette technique d'agrégation utilise les notions de "période d'insuffisance" d'un tronçon qui est la période de retour d'une pluie pour laquelle ce tronçon dépasse un certain seuil de dysfonctionnement (dans notre cas, le débordement) et de sensibilité du tissu urbain à un dysfonctionnement hydraulique du réseau. Cette dernière notion est nécessaire car certains tronçons peuvent très bien déborder sans induire de désordres apparents s'ils ont, par exemple, une capacité d'écoulement faible et/ou s'ils se trouvent dans une zone non bâtie. Les informations nécessaires à l'utilisation de cette méthode étant souvent de qualité inégale en termes de précision, nous avons pris le parti de raisonner non sur des valeurs déterminées ais sur des classes d'appartenance modélisées sous forme de sous-ensembles flous. 


\title{
Une approche pour la comparaison, du point de vue fonctionnement hydraulique, de propositions d'extension d'un réseau d'assainissement
}

\author{
An approach to compare, from the hydraulic operation \\ viewpoint, propositions of extension of an urban storm \\ drainage network
}

\author{
0. BLANPAIN ${ }^{*}$, A. KARNIB ${ }^{1}$, J. AL-HAJJAR ${ }^{1}$ et D. BOISSIER ${ }^{2}$
}

Reçu le 31 décembre 1996, accepté le 22 janvier 1998**.

SUMMARY

Software packages developed for the design of urban storm drainage networks allow several solutions to be proposed for the same problem. It then falls to the designer to choose which solution to use, the main problem being the evaluation of the efficiency (quality) of each solution. A multi-criteria approach represents one theoretical solution to the problem. This necessitates the determination of which criteria to use and how to evaluate them. In this paper, we present a method of evaluation of the criteria related directly to the functioning of an urban storm drainage network after its extension. This method is developed on the basis of hydraulic simulations of the network. These simulations produce results (histograms of discharge, water levels, rates of filling, hydraulic head,...) for each pipe. Given these results, the designer must be able to assess whether the proposed solution is satisfactory, and then compare it with other solutions. The problem is therefore to be able to evaluate a complete network, whereas the results of conventional simulations present a fragmented and partial view of its functioning (pipe by pipe). A solution to this problem is proposed in the form of a tool, able to calculate a single combined value from the simulation results. The following calculation steps are proposed: First we model the effectiveness of each pipe. To do that, we determine the "return period of failure" of a pipe which is the return period of a rainfall for which the pipe passes a certain level of failure (in this paper, we take the level of failure as the state of overflow). The rainfall model used is the same one used for the design of the network. Then we attribute a numerical value $(S)$ for the operation of a pipe according to its return period of failure by way of a satisfaction function. Secondly we model the weighting given to each pipe. This weighting is constructed from the discharge capacity of the pipe and the sensitivity of the urban fabric (in proximity to the pipe) to system failure. The

1. LAMH, Technoparc futura, Université d'Artois, 62400 Béthune, France, E-mail : blanpain@univ-artois.fr.

2. LERMES, CUST, Université Blaise Pascal, BP 206, 63174 Aubière, France.

Communication présentée au Colloque International du GRUTTEE, les 19 et 20 mars 1997 à Rennes.

* Correspondance. e-mail : blanpain@univ-artois.tr.

* * Les commentaires seront reçus jusqu'au 31 décembre 1998. 
discharge capacity is calculated using Manning's formula on the basis of diameter, slope and internal roughness. The value of the coefficient $(R)$, which indicates the sensitivity, necessitates a good knowledge of the urban fabric. Among the important variables related to this factor, we can identify the population density, the traffic density and the density of land use -DLU- (this variable is identified by the density of residential land use, the density of commercial land use,...). We can then write $R=f($ density of population, density of traffic, DLU,...). Considering the difficulty of the identification of (f), we preferred to explore an expert approach. The rules have been identified from a bibliographical analysis and limited expertise. An example of theses rules is presented here: IF density of population is high and density of commercial land use is average THEN the degree of sensibility is average. The examination of the identified rules shows the use of words like low, average and high. To model this linguistic qualifying information, we have chosen fuzzy sets. Also the inferences of fuzzy information are treated by using operations of fuzzy logic. Finally, we aggregate the results with the following equation: $C=\sum_{i=1}^{n} Q_{a 1} \cdot R_{i} \cdot \Delta S_{i}$, where $D S_{i}=S_{i}-S_{i}$ ' represents a measure of the effect of network modification upon the operation of the pipe $i\left(S_{i}\right.$ and $S_{i}$ ' are the effectiveness of the pipe respectively before and after the proposed modification), $Q_{a i}$ is the discharge capacity of the pipe $i, R_{i}$ is the coefficient of sensitivity of the area to failure associated with pipe $i$ and $C$ is a factor which quantifies the effect on the general operation of the network. With the coefficient $\mathrm{C}$, the designer is now able to classify the different solutions of extension of an existing urban drainage network according to their impacts on its functioning and to introduce this classification order in a multi-criteria method.

Key-words: urban storm drainage, hydraulic operation, fuzzy inferences, evaluation.

\section{RÉSUMÉ}

Les outils informatiques développés dans le cadre de la conception des réseaux d'assainissement permettent de concevoir plusieurs solutions de réseaux pour un même problème. Il revient ensuite au décideur de choisir quelle solution retenir. Le problème qui se pose alors est la comparaison des propositions selon des critères économiques, écologiques, de fonctionnement, de maintenance,... La difficulté vient du fait que certains de ces critères sont difficiles à évaluer. Nous présentons dans cet article une méthode permettant d'évaluer l'un de ces critères : le fonctionnement global d'un réseau d'assainissement suite à l'extension de ce dernier. Cette méthode est basée sur des simulations hydrauliques. Or ces simulations donnent des informations en chaque noud du réseau (histogrammes de vitesses, débits ou hauteurs de charge) et non une indication sur la qualité de fonctionnement du réseau dans son ensemble. Il nous a donc fallu élaborer une technique d'agrégation permettant de passer de l'élément isolé (le tronçon) à l'ensemble organisé (le réseau). Cette technique d'agrégation utilise les notions de «période d'insuffisance * d'un tronçon qui est la période de retour d'une pluie pour laquelle ce tronçon dépasse un certain seuil de dysfonctionnement (dans notre cas, le débordement) et de sensibilité du tissu urbain à un dysfonctionnement hydraulique du réseau. Cette dernière notion est nécessaire car certains tronçons peuvent très bien déborder sans induire de désordres apparents s'ils ont, par exemple, une capacité d'écoulement faible et/ou s'ils se trouvent dans une zone non bâtie. Les informations nécessaires à l'utilisation de cette méthode étant souvent de qualité inégale en termes de précision, nous avons pris le parti de raisonner non sur des valeurs déterminées mais sur des classes d'appartenance modélisées sous forme de sous-ensembles flous.

Mots clés : assainissement pluvial, fonctionnement hydraulique, inférences floues, évaluation. 


\section{1 - POSITIONNEMENT DE L'ÉTUDE}

Afin d'augmenter l'efficacité de leur réseau d'assainissement (objectifs écologiques et sanitaires) et d'optimiser les investissements (objectif économique), de plus en plus de collectivités locales se dotent de logiciels techniques d'assainissement. Ces outils, qui ont déjà démontré leur utilité et leur efficacité, permettent la conception rapide de plusieurs propositions de solutions à un problème donné. Néanmoins, les concepteurs ne disposent pas d'outils de comparaison des solutions entre elles pour guider le choix du décideur (KIM, 1990) (KUBA et al., 1996). Pour aider à cette comparaison, l'approche multicritère (ROY et BOUYSSOU, 1993) est une voie de réponse. Sa mise en œuvre nécessite de déterminer quels sont les critères à utiliser, comment ils peuvent être évalués et ensuite de définir comment les solutions peuvent être comparées (VINCKE, 1989).

Dans cet article, nous nous plaçons dans une optique d'aide à la décision. Nous étudions le cas d'une extension d'un réseau et nous nous limitons à l'évaluation d'un critère lié au fonctionnement hydraulique du réseau. Nous ne discuterons pas de la validité de la représentation hydrologique et hydraulique utilisée pour le problème. En effet, même si il existe de nombreuses sources d'imprécisions, d'inexactitudes et d'erreurs possibles dans les modélisations des objets et phénomènes étudiés, le décideur est bien obligé, afin d'effectuer son choix, d'émettre l'hypothèse que la représentation retenue est, sinon exacte, au minimum adéquate. L'exposé du problème est alors le suivant :

Soit un réseau existant, soient plusieurs propositions d'extension de ce réseau, quelle est l'extension qui permet d'avoir le meilleur fonctionnement du réseau complet?

Le problème peut se décomposer en 3 sous-problèmes :

P1 - Comment apprécier la qualité élémentaire de fonctionnement d'un tronçon?

P2 - Comment apprécier la qualité globale de fonctionnement d'un réseau à partir de la qualité élémentaire de chacun de ses tronçons?

P3 - Comment comparer chaque proposition d'extension vis-à-vis de cette qualité globale?

En ce qui concerne l'appréciation de la qualité élémentaire de fonctionnement d'un tronçon (problème $\mathrm{P} 1$ ), il est, au minimum, nécessaire de pouvoir comparer les débits susceptibles de transiter dans le tronçon considéré et son débit capable. L'appréciation pourra alors porter sur la période de retour du dépassement de cette capacité (STU, 1989). Cette appréciation n'est cependant pas toujours suffisante. En effet, le critère pris en compte n'est pas forcément significatif pour mesurer la qualité ou la non-qualité du fonctionnement. Par exemple, certains tronçons peuvent très bien déborder sans induire de désordres apparents s'ils ont, par exemple, une capacité d'écoulement faible et/ou s'ils se trouvent dans une zone non bâtie. Il est donc nécessaire, pour établir la qualité de fonctionnement d'un réseau, de tenir compte de la capacité des tronçons et des incidences probables d'un dépassement de capacité.

Dans le paragraphe $\S 2$, nous décrivons l'approche proposée pour construire le critère "répercussions globales sur l'existant". Dans le paragraphe $\$ 2.2$, nous présentons une méthode d'évaluation de la qualité de fonctionnement d'un tronçon. La prise en compte des incidences éventuelles d'un dépassement de 
capacité nécessite la détermination de la sensibilité du tissu urbain à un dysfonctionnement du réseau. Ceci est examiné au paragraphe $\$ 2.3$. La détermination finale du critère " répercussions globales sur l'existant » fait l'objet du paragraphe $\S 2.4$. Enfin nous montrons le bien fondé de la démarche par un exemple d'application sur un cas fictif $(\$ 3)$.

\section{2 - PROPOSITION D'UNE MÉTHODE DE CONSTRUCTION DU CRITÈRE “ RÉPERCUSSIONS GLOBALES SUR L'EXISTANT »}

\subsection{Définitions}

Nous définissons le critère "répercussions élémentaires sur l'existant" comme étant le critère associé aux conséquences éventuelles d'une extension du réseau. Cette répercussion est liée à un tronçon ("élémentaire "). Celle-ci se traduit par une modification de débit dans le tronçon. Cette modification pourra entraîner, le cas échéant, des dysfonctionnements et des dégradations de la zone de tissu urbain associée au tronçon.

Le critère " répercussions globales sur l'existant " représente les conséquences éventuelles de l'extension du réseau sur tout le tissu urbain associé au réseau. Ce critère global est défini par l'agrégation des critères élémentaires. La part que prend un critère élémentaire dans le critère global est liée à :

- limportance du débit de chaque tronçon. En effet, un tronçon défaillant véhiculant un débit faible causera peu de dégâts par rapport à un tronçon défaillant de grand débit ;

- la sensibilité du tissu urbain à un dysfonctionnement du tronçon. L'environnement d'un tronçon défaillant est capital pour l'estimation de l'importance des dégâts (à titre d'exemple un débordement dans une zone inhabitée n'aura pas les mêmes conséquences qu'un débordement dans le centre-ville!).

\subsection{Répercussions élémentaires sur l'existant}

Pour donner une note de qualité à chaque tronçon, nous proposons deux étapes. La première est de déterminer la période d'insuffisance du tronçon et la seconde est de formuler la note de qualité du tronçon suivant sa période d'insuffisance.

\subsubsection{Détermination de la période d'insuffisance}

Nous appelons "période d'insuffisance " la période de retour d'une pluie pour laquelle le tronçon dépasse un certain seuil de dysfonctionnement. Comme seuil de dysfonctionnement, nous avons choisi le paramètre "hauteur de débordement ". Nous choisissons ce paramètre pour les raisons suivantes: tous les logiciels techniques d'assainissement permettent d'obtenir cette donnée et, même s'il existe des modèles permettant de simuler de manière plus complète les débordements, ces modèles nécessitent des données rarement disponibles. Nous identifions alors deux états de fonctionnement :

Soient $\mathrm{Hc}$ la hauteur de charge donnée par un modèle de simulation hydraulique et $\mathrm{H}$ la profondeur du tronçon par rapport au niveau du terrain naturel, alors 
nous avons l'état 1 si $\mathrm{Hc}<\mathrm{H}$ (pas de débordement) et l'état 2 si $\mathrm{Hc}>\mathrm{H}$ (débordement en surface).

Pour déterminer la période d'insuffisance de chaque tronçon, nous utilisons l'algorithme suivant (figure 1):

- choix du type de pluie de projet : les pluies envisagées correspondent à des périodes de retour de 1 an, 2 ans, 3 ans, 4 ans, 5 ans, 10 ans, 15 ans, 20 ans, 25 ans, 30 ans, 40 ans, 50 ans,

- simulation hydraulique,

- détermination des tronçons qui dépassent le seuil de dysfonctionnement choisi.

Cette démarche est répétée pour les différentes pluies envisagées, et nous permet alors de déterminer la période d'insuffisance de chaque tronçon. Dans la figure 1 , j est le nombre de tronçons composant le réseau, i le nombre de périodes de retour de pluie utilisées, $T_{i}$ le nom du jème tronçon, $P_{i}$ la valeur de période de retour de la ième pluie et $T_{j}{ }_{j}$ le nom des tronçons dépassant le seuil de dysfonctionnement pour la jème pluie.

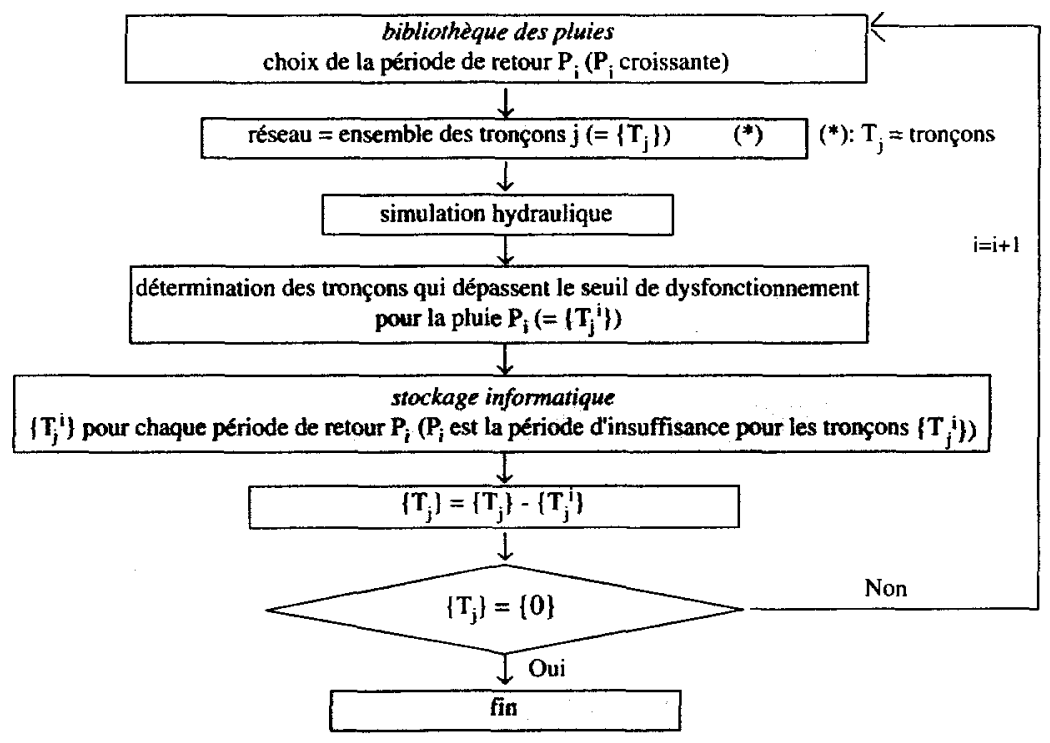

Figure 1 Algorithme de la détermination des périodes d'insuffisance des tronçons.

Determination of the return period of failure algorithm.

\subsubsection{Détermination de la note de qualité du tronçon suivant sa période d'insuffisance}

Pour déterminer cette note $S_{i}$, nous utilisons une fonction de satisfaction $S$ qui croît avec la période d'insuffisance et qui peut par exemple prendre la forme linéaire de la figure 2.

Pour la paramétrer, nous proposons une période de retour de 50 ans comme période de satisfaction totale et une période inférieure à 1 an pour période de satisfaction nulle. 


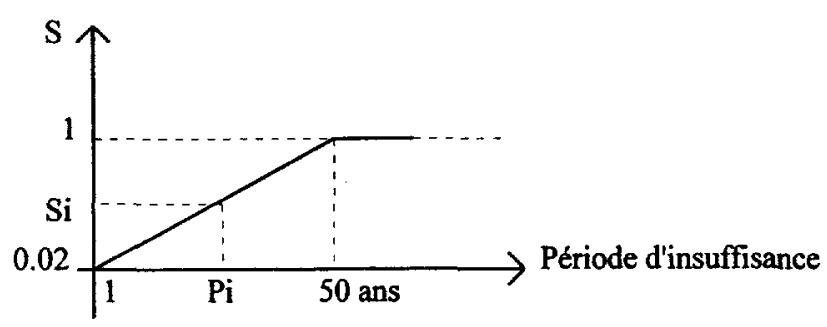

Figure 2 Fonction de satisfaction liée à la période d'insuffisance d'un tronçon.

Degree of satisfaction relative to the function of the return period of a pipe failure.

La procédure de qualification de chaque tronçon est réalisée plusieurs fois : une fois avant l'extension du réseau et ensuite une fois pour chaque proposition d'extension.

\subsection{Détermination du degré de sensibilité du tissu urbain à un dysfonctionnement hydraulique}

\subsubsection{Généralités}

Nous appelons « zone de conséquences » la zone concernée par le dysfonctionnement d'un tronçon. Le degré de sensibilité $R_{\mathrm{i}}$ représente la sensibilité de "la zone de conséquences " au dysfonctionnement du tronçon i. La détermination de $R_{i}$ est fonction des paramètres suivants :

- la densité de population (nombre des personnes par hectare),

- la densité de trafic (nombre des véhicules par jour),

- les coefficients d'utilisation du sol (CUS), et $R_{i}=f$ (densité de population, densité de trafic, CUS)

(Les considérations qui ont guidé notre choix de paramètres sont, avant tout, pragmatiques : il s'agit de données généralement disponibles)

Devant la difficulté d'identification de la fonction $f$ (ROGER JANG et GULLEY, 1995), nous avons préféré l'exploration d'une approche « système à base d'inférence floue $"$.

\subsubsection{Les règles}

Le problème rencontré a été formulé sous forme de règles mettant en jeux les différents paramètres déjà énumérés : la densité de population, la densité du trafic et les coefficients d'utilisation du sol. Ces règles issues d'une extraction d'expertise (études de cas et interviews) se présentent sous la forme suivante :

Sl la densité de population est haute et le coefficient d'utilisation du sol commercial est moyen ALORS le degré de sensibilité est haut

Les variables dans les prémisses et les conclusions ont des attributs qualitatifs et imprécis que nous modélisons par des sous-ensembles flous.

\subsubsection{Les sous-ensembles flous (SEF)}

La théorie des sous-ensembles flous (ZADEH, 1978) permet la prise en compte d'informations déclarées par des qualificatifs imprécis (haut, moyen...). 
Les sous-ensembles flous utilisés sont de type trapézoïdal. Dans ce mode de représentation, la fonction d'appartenance est définie à partir de 5 paramètres ( $m, n, \alpha, \beta, h$ ) et deux fonctions $L$ (left) et $R$ (right) (figure 3).

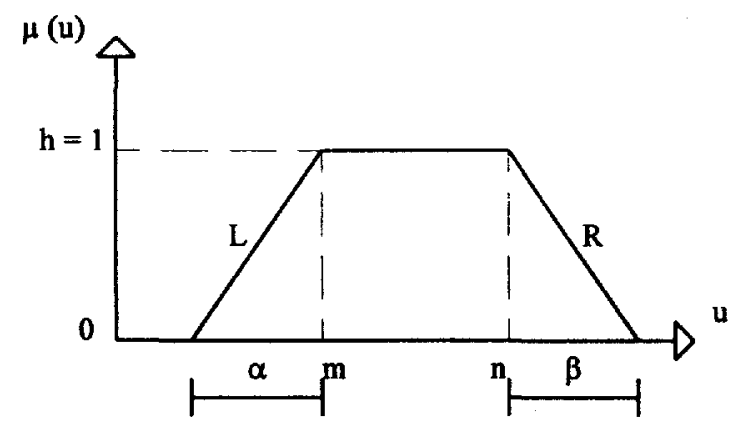

Figure 3 SEF de type trapèze.

Fuzzy set of trapezoidal type.

Cette fonction d'appartenance que nous associons à une variable est fonction de la nature de la variable. Elle prend la forme d'une série de trapèzes se coupant en un point dont l'ordonnée représente le degré d'appartenance des limites numériques à deux niveaux contigus. Une valeur $\mu(u)$ de 1 indique une appartenance totale à la classe envisagée, une valeur de 0 indique une exclusion totale, une valeur comprise entre 0 et 1 indique une appartenance partielle. Nous présentons ci-dessous (figure 4), le cas de la variable « densité de population ».

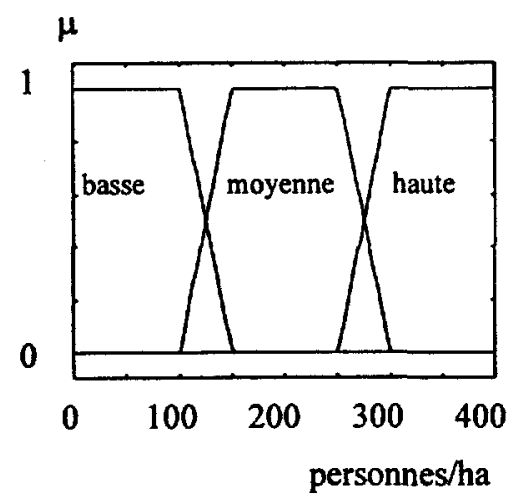

Figure 4 Modélisation de la variable densité de population (personnes/ha).

Density of population (persons/ha).

\subsubsection{L'intérence floue}

Nous avons déjà fait état de règles floues de la forme (cf. 2.3.2.) : « Si X est $A$ alors $Y$ est $B$ " où $X$ et $Y$ sont deux variables linguistiques, alors que $A$ et $B$ sont deuX appréciations imprécises. 
Le but de l'inférence floue (ou évaluation des règles) est de déterminer les sorties du système à partir des entrées floues issues de la fuzzification des entrées réelles et des règles floues (TONG TONG, 1995) (figure 5).

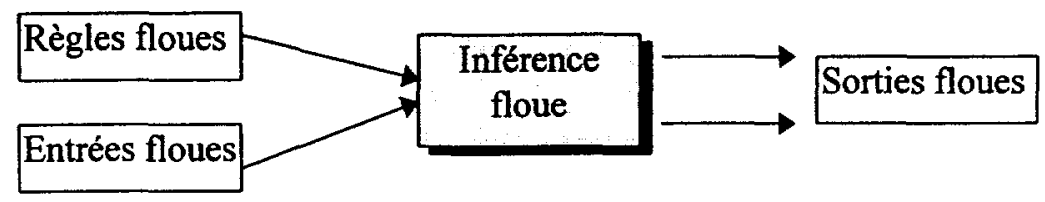

Figure 5 Illustration de l'inférence floue.

Schematic diagram of the fuzzy inference.

Il existe différentes méthodes d'inférences floues (TONG TONG, 1995), nous avons choisi une méthode pratique et simple : celle de Mamdani (MAMDANI, 1976) (MAMDANI et ASSILIAN, 1975).

\subsubsection{Description du système d'inférence floue utilisé pour la détermination de $\mathbf{R}$}

La production de la conclusion du système passe par les étapes suivantes :

1) Fuzzification des entrées: La première étape consiste à appliquer les variables d'entrée aux sous-ensembles flous prédéfinis dans le but de déterminer les degrés d'appartenance.

2) Application des opérateurs flous et de la méthode de l'implication : l'entrée de cette étape est l'ensemble des degrés d'appartenance déterminés dans la première étape. Elle consiste à déterminer les conclusions des règles (une par une) en fonction des degrés d'appartenance trouvés dans la première étape.

3) Agrégation : Cette étape consiste à agréger dans un seul sous-ensemble flou tous les sous-ensembles flous qui résultent de l'étape précédente.

Ces étapes sont illustrées par l'exemple de la figure 6 construit à partir de deux règles.

Le système a été implémenté dans l'environnement du logiciel "MATLAB " (ROGER JANG et GULLEY, 1995). La structure de base de ce système est représentée sur la figure 7.

\subsubsection{Utilisation de la procédure}

Pour calculer $R_{i}$ pour un tronçon par notre système, il faut suivre les étapes suivantes:

1) Identifier la « zone de conséquences » du tronçon.

2) Calculer les variables d'entrée dans la "zone des conséquences " du tronçon.

3) Appliquer les valeurs obtenues dans le système de détermination de $R_{i}$.

\subsubsection{Limites et hypothèses}

Chaque ville est unique par sa structure, sa topographie et sa richesse. Nous avons proposé une méthode qui permet d'évaluer le coefficient de sensibilité $\mathbf{R}_{\mathrm{i}}$, or cette méthode nécessite suivant chaque ville de :

- déterminer les bornes numériques des variables linguistiques ;

- déterminer la zone des conséquences de chaque tronçon. 


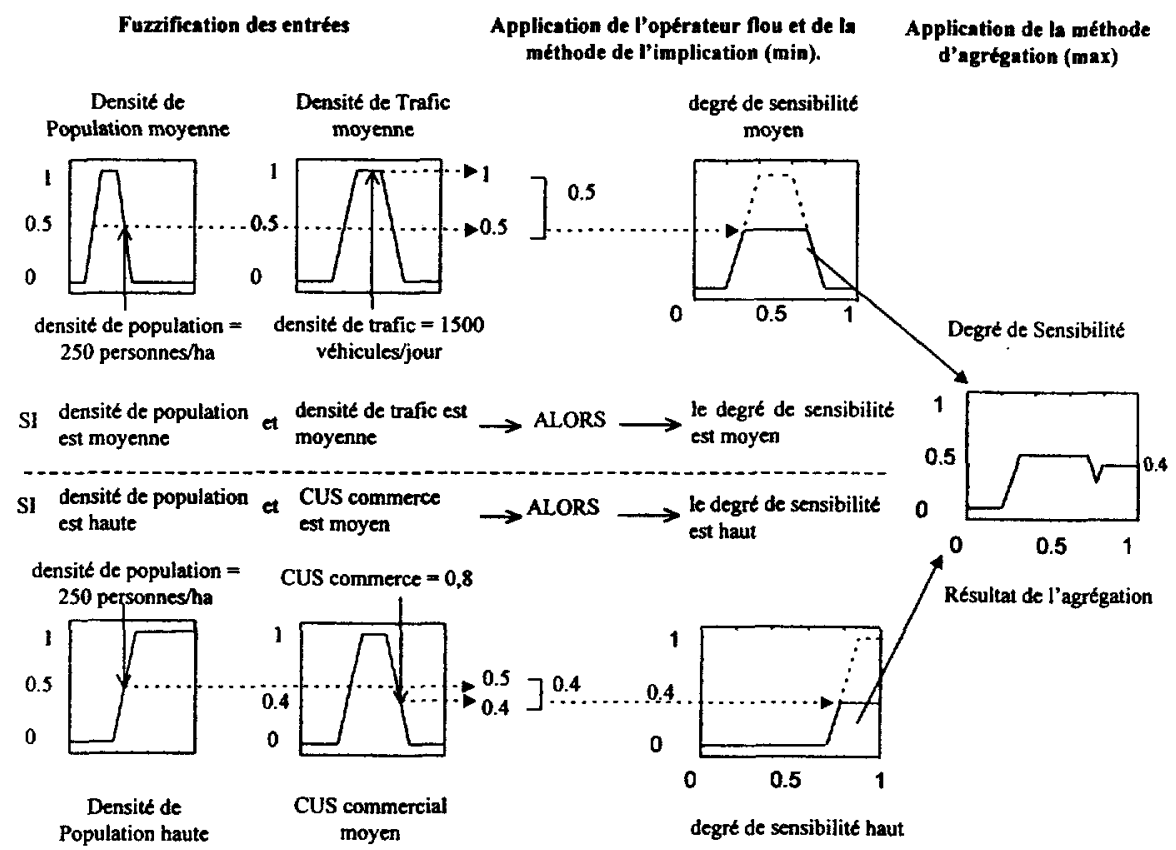

Figure 6 lllustration des différentes étapes du système de détermination de $R_{i}$.

Illustration of the different steps of the Ri determination system.

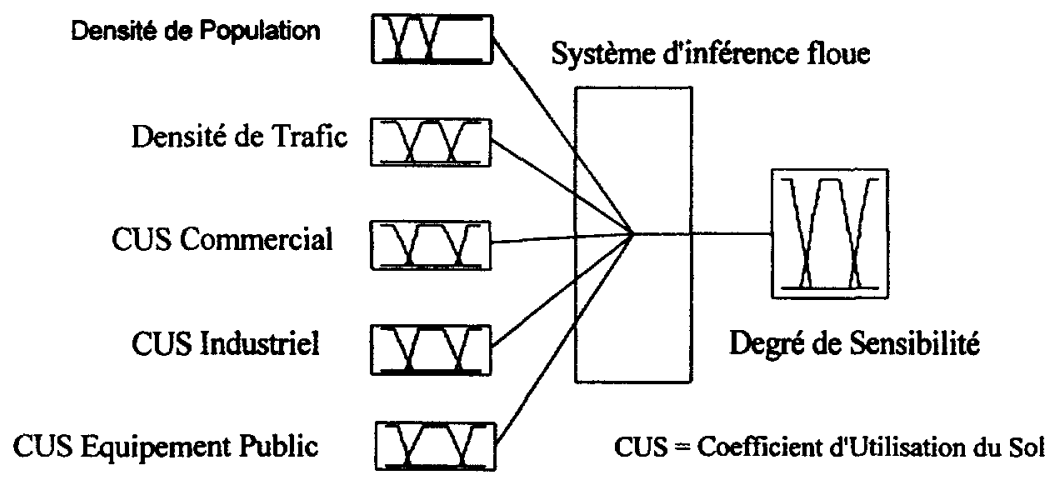

Figure 7 Structure de base du système d'inférence floue.

Basic structure of the fuzzy inference system.

Dans l'état actuel des connaissances, il ne nous est pas possible de concevoir un outil permettant la détermination automatique des informations nécessaires à l'évaluation du coefficient de sensibilité $\mathbf{R}_{\mathrm{i}}$. Cette tâche reste, pour l'instant, du ressort des ingénieurs gestionnaires du réseau. Leur expérience du terrain devrait leur permettre de mener à bien la détermination des paramètres nécessaires à la méthode proposée. 


\subsection{Critère “ Répercussions globales sur l'existant ", C}

Après avoir évaluer les critères * Répercussions élémentaires sur l'existant " avant et après extension notés $S_{i}$ et $S_{i}^{\prime}$ respectivement (cf. 2.2.2.), nous calculons pour chaque tronçon les écarts $\Delta S_{i}=S_{i}-S_{i}$.

Pour évaluer le critère «Répercussions globales sur l'existant », C, nous proposons la formule suivante :

$$
C=\sum_{i=1}^{n} Q_{a i} \cdot R_{i} \cdot \Delta S_{i}
$$

Où :

- C est un facteur qui représente le degré de répercussion sur le fonctionnement global du réseau. L'échelle de préférence accordée à ce facteur est le suivant : plus la valeur de $\mathrm{C}$ est importante, moins la solution pour ce critère est bonne.

- $R_{\mathrm{i}}$ et $\mathrm{Q}_{\mathrm{ai}}$ permettent de prendre en compte l'importance des tronçons par rapport au réseau. Le premier poids est lié à la sensibilité du tissu urbain à un dysfonctionnement (cf. 2.3).

Le deuxième est lié à l'importance du tronçon par rapport au fonctionnement du réseau, ce poids sera le débit capable de chaque tronçon, il est calculé par la formule de Manning.

Le facteur $C$ doit être évalué pour chaque proposition d'extension du réseau, ce qui nous permet par la suite d'introduire les évaluations des différentes propositions dans un système d'aide à la décision pour choisir ou classer les meilleures solutions (KARNIB, 1996).

\section{3 - EXEMPLE D'APPLICATION SUR UN CAS FICTIF}

Dans cet exemple d'application, nous allons examiner quatre propositions de réseau d'assainissement d'eau pluviale pour l'aménagement d'une zone NA dont la superficie est de 25 ha. Cette zone se trouve à l'amont d'une commune dont la superficie est de 350 ha. La figure 8 présente le réseau existant avec les différentes propositions d'aménagement.

La variante $\mathrm{A} 1$ est un réseau sans maille moyennement ramifié ; la variante $A 2$ est le même réseau que $A 1$ mais avec la présence d'un bassin de rétention, la variante $A 3$ est un réseau maillé et la variante $A 4$ est le même réseau que $A 3$ à la différence près qu'il est connecté au réseau existant par deux nœuds. Nous développerons dans ce qui suit la détermination du critère "Répercussions globales sur l'existant » à travers la proposition A1. Le réseau de la zone NA a été conçu avec une pluie de projet de période de retour égale à 10 ans. Nous avons pris comme seuil de dysfonctionnement la hauteur de débordement, et comme période de retour maximale 50 ans.

Note: En ce qui concerne le modèle de pluie de projet utilisé, nous avons choisi un modèle de pluie de projet d'utilisation courante en France et facile à 

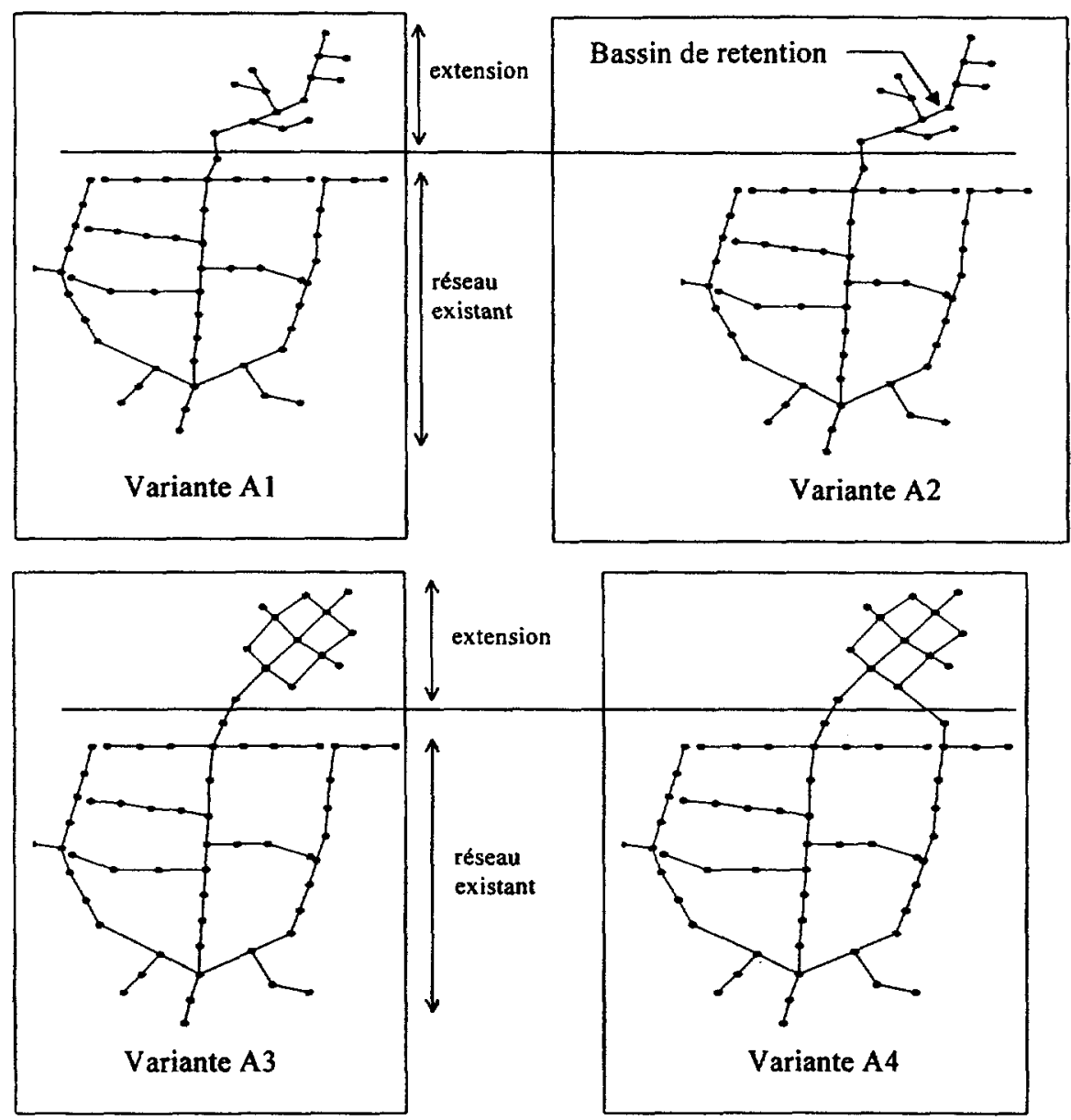

Figure 8 Les différentes propositions d'extension du réseau existant.

Simplified plan of the network with its different proposals of extension.

mettre en cuvre : le modèle développé à l'INSA de Lyon par B. Chocat (STU, 1989). Tout autre modèle de pluie est utilisable à condition d'être représentatif de la pluviométrie locale.

Suite aux simulations hydrauliques effectuées avec le logiciel CEDRE (CHOCAT, 1990), nous avons obtenu les résultats suivants (tableau 1). Dans ce tableau, nous présentons seulement les tronçons où l'écart $\Delta S_{i}=S_{i}-S_{i}^{\prime} \neq 0$.

Pour pouvoir évaluer le degré de répercussions $C$ de la proposition d'extension sur le réseau existant, nous calculons le coefficient de sensibilité du tissu urbain $R_{i}$ pour les tronçons $1,2,9,12,13$ et 16 .

Les bornes numériques des variables d'entrée utilisées dans le système sont définies par le projeteur. En consultant les plans topographiques de la ville, il définit pour chaque tronçon sa «zone de conséquences". Le projeteur déclare pour chaque zone les variables d'entrée. 
Tableau 1 Résultat de simulations hydrologiques sur le réseau.

Table 1 Hydraulic simulation results on the network.

\begin{tabular}{|c|c|c|c|c|c|c|}
\hline \multirow[b]{2}{*}{ Tronçan } & \multicolumn{2}{|c|}{ Réseau existant } & \multicolumn{2}{|c|}{ Réseau moditié } & \multirow[b]{2}{*}{$\Delta \mathbf{S}_{1}$} & \multirow[b]{2}{*}{$a_{a i} m^{3} / s e c$} \\
\hline & $\begin{array}{c}\text { Période } \\
\text { d'insulfisance }\end{array}$ & $s_{i}$ & $\begin{array}{c}\text { Période } \\
\text { d'insutlisance }\end{array}$ & $S_{i}^{\prime}$ & & \\
\hline 1 & 15 ans & 0,3 & 5 ans & 0,1 & 0,2 & 17,5 \\
\hline 2 & 15 ans & 0,3 & 4 ans & 0,08 & 0,22 & 17,5 \\
\hline 9 & $>50$ ans & 1 & 30 ans & 0,6 & 0,4 & 14,1 \\
\hline 12 & $>50$ ans & 1 & 30 ans & 0,6 & 0,4 & 14,1 \\
\hline 13 & $>50$ ans & 1 & 40 ans & 0,8 & 0,2 & 14,1 \\
\hline 16 & $>50$ ans & 1 & 40 ans & 0,8 . & 0,2 & 14,1 \\
\hline
\end{tabular}

À partir de ces informations et en appliquant le système décrit précédemment, $\mathrm{A}_{\mathrm{i}}$ est calculé pour chaque tronçon. Les résultats sont présentés dans le tableau 2.

Tableau 2 Résultats obtenus pour les tronçons défaillants.

Table 2 Results obtained for failing pipes.

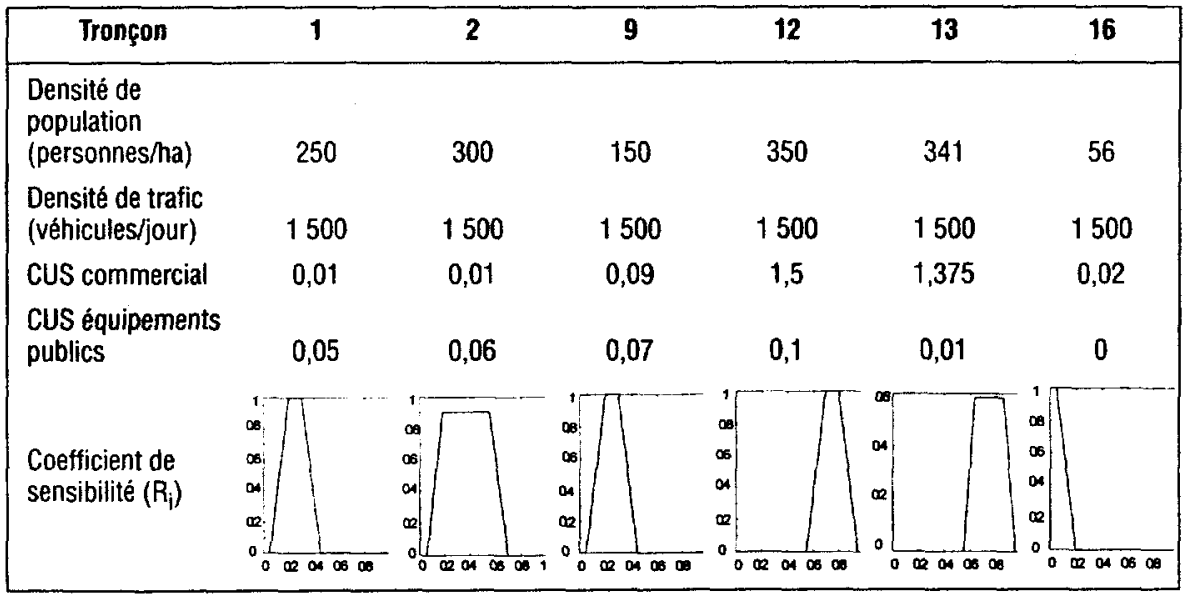

Nous calculons ensuite le facteur $\mathrm{C}$, à partir de la formule (2). Pour les quatre propositions d'extension du réseau existant, nous obtenons les valeurs floues de C suivantes (figure 9).

II reste alors à comparer les différents sous-ensembles flous entre eux. Diverses méthodes de comparaison existent (DUBOIS et PRADE, 1983) (KARNIB, 1996). Pour notre exemple, avec la méthode PREFUN (KARNIB et al., 1997) qui utilise la comparaison des intervalles obtenus par $\alpha$-coupes, nous obtenons les valeurs de surclassement suivantes : $S(A 1, A 2)=0, S(A 1, A 3)=0,5, S(A 1, A 4)=0,1, S(A 2$, $A 1)=1, S(A 2, A 3)=1, S(A 2, A 4)=1, S(A 3, A 1)=0,5, S(A 3, A 2)=0, S(A 3, A 4)$ $=0,1, S(A 4, A 1)=0,567, S(A 4, A 2)=0, S(A 4, A 3)=0,576$. Plus la valeur est grande, plus le premier terme surclasse le second. Ces valeurs sont directement utilisables dans une méthode d'analyse multicritère utilisant la relation de surclassement telle que ELECTRE (ROY et HUGONNARD, 1982). 


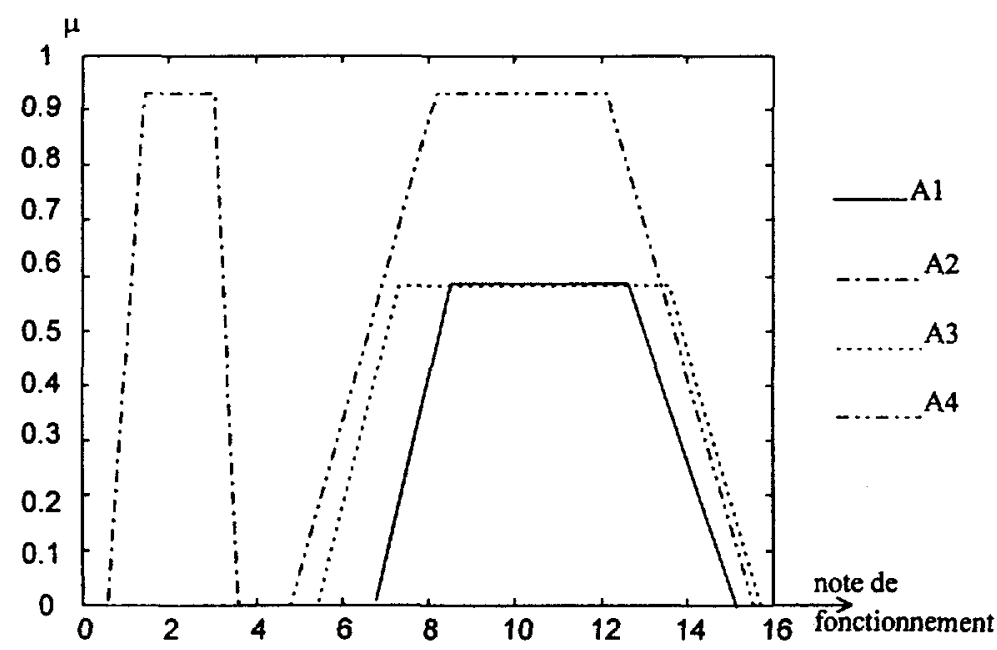

Figure 9 Le facteur $\mathrm{C}$ pour les propositions d'extensions $\mathrm{A} 1, \mathrm{~A} 2, \mathrm{~A} 3$ et $\mathrm{A} 4$.

Evaluation of the $C$ factor for the proposals of extension $A 1, A 2, A 3$ and $A 4$.

\section{4- CONCLUSIONS}

Parmi tous les critères nécessaires à la comparaison de propositions d'extension d'un réseau d'assainissement, nous avons choisi de développer ici la présentation du critère " Répercussions globales sur l'existant ". Ce critère prend en compte le comportement hydraulique de chaque tronçon par le biais de la notion d'insuffisance. II prend aussi en compte les conséquences d'un dysfonctionnement par le biais de la notion de degré de sensibilité $\mathbf{R}_{\mathrm{i}}$.

Lapport fondamental réside ici dans l'introduction de qualitatif et d'imprécis caractérisant " la zone de conséquences " sous forme de sous-ensembles flous. Cette modélisation nécessite alors l'utilisation d'inférences floues.

Un autre apport est la caractérisation globale de la qualité de l'extension par agrégation des critères associés à chaque tronçon. Le facteur $C$ évalué pour chaque proposition d'extension du réseau nous permet d'introduire les évaluations des différentes propositions dans un système d'aide à la décision pour choisir ou classer les meilleures solutions (KARNIB, 1996).

Enfin, cette méthode a été appliquée sur un cas réel dans la ville d'Annequin dans le nord de la France (KARNIB, 1996). II s'agissait de corriger le réseau d'assainissement qui présentait des dysfonctionnements chroniques en certains points (débordements, inondations de caves) et de faire en sorte qu'il réponde aux futures normes européennes en ce qui concerne les rejets au milieu naturel. Trois solutions de restructuration ont été proposées par un bureau d'études. Les critères de choix retenus étaient le coût, le fonctionnement hydraulique (caractérisé par la méthode présentée dans cet article), les volumes d'eau rejetés par les déversoirs d'orage et l'esthétisme (ce dernier critère concerne l'implantation des bassins de rétention dans le tissu urbain). Suite à l'analyse multicritère effectuée avec la méthode ELECTRE IV (ROY et HUGONNARD, 1982), la mairie d'Annequin a 
choisi la solution classée en deuxième place. L'analyse de ce choix nous a montré qu'il correspondait à une diminution de l'importance du critère « volumes rejetés au milieu extérieur " et à une augmentation de celle du coût. Nous pouvons retenir de cette étude que, même si la solution classée en premier n'a pas été retenue, l'utilisation de la méthode d'analyse multicritère a poussé le décideur (en l'occurrence le maire) à réfléchir sur la base d'un rapport qualité/coût et non sur la seule base du coût (pratique courante, voir même majoritaire). Nous avons aussi remarqué lors de cette étude qu'il était nécessaire d'intégrer les décideurs lors de la construction des sous-ensembles flous associés aux zones de conséquences. La construction de ces sous-ensembles flous est en effet une composante à part entière de l'aide à la décision et y faire participer les décideurs permet de mieux leur faire accepter l'outil proposé.

\section{REMERCIEMENTS}

Cette étude a pu être menée grâce au soutien de la Communauté du Béthunois, de la Région Nord - Pas-de-Calais et de la Compagnie Générale des Eaux.

\section{RÉFÉRENCES BIBLIOGRAPHIQUES}

CHOCAT B., 1990. Logiciel CEDRE version 2.0 - Manuel de références, INSA de Lyon, Laboratoire méthodes, $307 \mathrm{p}$.

DUBOIS D., PRADE H., 1983, Ranking fuzzy numbers in the setting of possibility theory, Information Sciences, vol. 30, p. 183-224.

KARNIB A., 1996. Approche multicritère pour l'aide au choix d'une solution de réseau technique urbain. Application à un réseau d'assainissement pluvial, thèse de doctorat, Université d'Artois, $164 \mathrm{p}$.

KARNIB A., AL-HAWJAR J., BLANPAIN O., BOISSIER D., 1997. A multicriteria approach to deal with uncertainty and fuzziness in the design of urban storm drainage network. International Conference on Rehabilitation and Development of Civil Engineering Infrastructure Systems'97, Beyrouth, Liban, p. 517-528.

KIM T.J., 1990. Expert systerns: applications to urban planning, Springer-Verlag, $268 \mathrm{p}$.

KUBA P., METELKA T., PLISKA Z., 1996. Impacts of the hydroinformatics technology on the way of master planning, Hydroinformatics'96, Balkema, p. 363-368.

MAMDANI E.H., ASSILIAN S., 1975. An Experiment in Linguistic Synthesis with a Fuzzy
Logic Controller, International Journal of Man-Machine Studies, vol. 7, p. 1-13.

MAMDANI E.H., 1976. Advances in the linguistic synthesis of fuzzy controllers, International Journal of Man-Machine Studies, vol. 8, p. 669-678.

ROGER JANG J.S., GULLEY N., 1995. FUzZY logic toolbox for use with Matlab, MathWorks Inc., $188 \mathrm{p}$.

ROY B., BOUYSSOU D., 1993. Aide Multicritère à la Décision : Méthodes et cas, Editions Economica, $695 \mathrm{p}$.

ROY B., HUGONNARD J.C., 1982. Le plan d'extension du métro en banlieue parisienne, un cas type d'analyse multicritère, Les cahiers Scientifiques de la Revue Transports, vol. 6, p. 77-108

STU, 1989. Mémento sur l'évacuation des eaux pluviales, Éditions La documentation française, $349 \mathrm{p}$.

TONG TONG J.R., 1995. La logique flove, Éditions Hermès, $160 \mathrm{p}$.

VINCKE Ph., 1989. L'aide multicritere à la décision, Éditions Ellipses, $179 \mathrm{p}$.

ZADEH L.A., 1978. Fuzzy sets as a basis for a theory of possibility, Fuzzy Sets and Systems, vol. 1, n० 1, p. 3-28. 